\title{
Thermo-acoustic Parameters of Tetrabutylammonium borate and Perchlorate in Non-aqueous Solvents
}

\author{
MANPREET KAUR ${ }^{*}$ \\ Department of Chemistry, DAV College, Sector-10, 160010, Chandigarh, India. \\ *Corresponding author E-mail: sohalmanpreetkaur@yahoo.in
}

http://dx.doi.org/10.13005/ojc/370330

(Received: May 09, 2021; Accepted: June 15, 2021)

\begin{abstract}
Interactions of electrolytes in a binary mixture can be determined by various techniques. Ultrasonic velocity measurements prove to be one of the important tools for measuring various acoustic properties at variable temperature. Thermo-acoustic parameters like Isentropic compressibility $\left(\mathrm{K}_{\mathrm{s}}\right)$, Acoustic impedance $(Z)$, Free volume $\left(V_{f}\right)$, Absorption coefficient $\left(A b_{\text {scoeff }}\right)$, Intermolecular free length $\left(L_{f}\right)$, Gibb's free energy $(\Delta G)$, Relaxation time $(\tau)$, Rao's constant $\left(R_{m}\right)$, Internal pressure $\left(\pi_{\mathrm{i}}\right)$, Wada's constant $(\mathrm{w})$, and Entropy $(\mathrm{H})$ for Tetrabutylammonium tetraphenylborate $\left(\mathrm{Bu}_{4} \mathrm{NBPh}_{4}\right)$ and Tetrabutylammonium perchlorate $\left(\mathrm{Bu}_{4} \mathrm{NClO}_{4}\right)$ was calculated using experimental ultrasonic velocities, viscosities and densities at three different temperatures (298K, $308 \mathrm{~K}$ and $318 \mathrm{~K})$ and 1 atmospheric pressure in non-aqueous solvents like Dimethylsulfoxide (DMSO), Pyridine (Py) and their binary mixtures at $0,20,40,60,80$ and $100 \mathrm{~mol} \%$ of $P y$ at variable temperatures ranging from $298 \mathrm{~K}$ to $318 \mathrm{~K}$. Both $\mathrm{Bu}_{4} \mathrm{NBPh}_{4}, \mathrm{Bu}_{4} \mathrm{NClO}_{4}$, showed an increase in the ultrasonic velocity values at all the temperatures. This shows that molecular interactions are taking place in both the electrolytes. These increases in the molecular interactions with increase in the concentration of electrolytes in the solvent mixture were discussed in terms of solvent structural effects. And results showed the greater molecular interaction in DMSO rich regions.
\end{abstract}

Keywords: Acoustic studies, Non-aqueous solvents, Molecular interaction, Ultrasonic velocity, Density.

\section{INTRODUCTION}

The structure and interactions of electrolytes in a binary mixture can be determined using a variety of approaches and spectroscopic techniques. X-ray crystallography, chromatography, NMR, EPR, vibration and Raman spectroscopy, neutron and light scattering, circular dichroism (CD), infrared spectroscopy, and ultrasonic velocity measurements are among them. Ultrasonic velocity measurements have been discovered to be the most important method in the investigation of structure and molecular interactions occurring in solutions among these techniques. Ultrasonic wave propagation in a material has become a basic test for determining its properties ${ }^{1}$. Many researchers have demonstrated the critical and fundamental function of molecular specifics of the solvent species in determining particular interactions, which are responsible for macroscopic thermodynamic and other associated properties in non-electrolyte solutions ${ }^{2,3}$. Materials are typically treated in fluid

This is an Open Access article licensed under a Creative Commons license: Attribution 4.0 International (CC- BY). Published by Oriental Scientific Publishing Company @ 2018

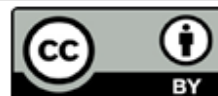


form in the chemical process industries, so their physical, chemical, and transport properties are essential ${ }^{4,5}$. The ultrasonic technique is a fast and non-destructive way to characterize materials ${ }^{6}$. The ultrasonic velocity in a liquid is fundamentally related to the binding forces between atoms or molecules, and it has been successfully used to characterize the physicochemical activity of liquid mixtures in the field of interactions and structural aspect studies ${ }^{7-9}$. The ultrasonic velocity, in combination with density and viscosity, provides a wealth of knowledge about ion interactions, dipoles, hydrogen bonding, multipolar and dispersive forces ${ }^{10}$. Since intermolecular and intramolecular association, complex formation, dipolar interactions, and related structural changes affect the system's compressibility, which causes corresponding variations in ultrasonic velocity, ultrasonic propagation parameters provide useful information about the behavior of liquid systems ${ }^{11}$. The various acoustic parameters are used to interpret the type and frequency of molecular interactions in the system ${ }^{12}$. Intermolecular interactions have an effect on the structural arrangement and form of molecules ${ }^{13,14}$. In the present studies thermo acoustic parameters like Isentropic compressibility $\left(\mathrm{K}_{\mathrm{s}}\right)$, Acoustic impedance $(\mathrm{Z})$, Free volume $\left(\mathrm{V}_{\mathrm{f}}\right)$, Absorption coefficient $\left(\mathrm{Ab}_{\mathrm{scoeff}}\right)$, Intermolecular free length $\left(L_{f}\right)$, Gibb's free energy $(\Delta \mathrm{G})$, Relaxation time $(\tau)$, Rao's constant $\left(\mathrm{R}_{\mathrm{m}}\right)$, Internal pressure $(\pi \mathrm{i})$, Wada's constant $(\mathrm{w})$, and Entropy $(\mathrm{H})$ for Tetrabutylammonium tetraphenylborate $\left(\mathrm{Bu}_{4} \mathrm{NBPh}_{4}\right)$ and Tetrabutylammonium perchlorate $\left(\mathrm{Bu}_{4} \mathrm{NClO}_{4}\right)$ was calculated using experimental velocities, viscosities and densities at three different temperatures (298K, $308 \mathrm{~K}$ and $318 \mathrm{~K}$ ) and 1 atmospheric pressure in nonaqueous solvents like Dimethylsulfoxide (DMSO), Pyridine (Py) and their binary mixtures at 0, 20, 40, 60,80 and $100 \mathrm{~mol} \%$ of Py at variable temperatures ranging from $298 \mathrm{~K}$ to $318 \mathrm{~K}$.

\section{MATERIALS AND METHODS}

\section{Apparatus}

Using experimental densities, the necessary proportion of solvents were mixed with the preliminary conversion of the required mass of each solvent to a volume at $298 \mathrm{~K}$. The solvents were mixable throughout. An electronic balance (SAG 285, Mettler Toledo) with a precision of $\pm 10^{-7}$ $\mathrm{kg}$ was used for the mass measurements. Each solvent were kept in an air tight container to avoid any kind of contamination. Density and ultrasonic velocity were measured by DSA 5000M from Anton Parr at 298K using long tube of borosilicate glass (U shaped). The sample was then excited to oscillate at its characteristic frequency of $2 \mathrm{MHz}$. Apparatus was calibrated at the beginning of every set of reading with distilled water and air. For calibration, the density \& ultrasonic velocity of known liquids like water $\left(\rho=0.99705 \mathrm{~g} \mathrm{~cm}^{-3}, \mathrm{u}=1496.68 \mathrm{~ms}^{-1}\right)$ and acetonitrile $\left(\rho=0.77687 \mathrm{~g} \mathrm{~cm}^{-3}, \mathrm{u}=1280.9 \mathrm{~ms}^{-1}\right)$ were measured at $298 \mathrm{~K}$ and it agreed well with literature values of $\rho=0.99707 \mathrm{~g} \mathrm{~cm}^{-3}{ }^{15}, \mathrm{u}=1496.6 \mathrm{~ms}^{-16}$ for water and $\rho=0.77685 \mathrm{~g} \mathrm{~cm}^{-3}, \mathrm{u}=1280.8 \mathrm{~ms}^{-1} 17$ for acetonitrile. Accuracy in the density value was $\pm 1 \mathrm{x}$ $10^{-5} \mathrm{~g} \cdot \mathrm{cm}^{-3}$ and for ultrasonic velocity was $\pm 0.1 \mathrm{~ms}^{-1}$. All the experiments were performed in temperature controlled DSA apparatus $( \pm 0.01 \mathrm{~K})$. Viscosity was measured by SV-10 viscometer (A\&D Co. Ltd.) at a frequency of $30 \mathrm{~Hz}$. The apparatus was calibrated prior every reading with distilled water. Each set of reading was repeated twice for better reproducibility of result. The accuracy in the viscosity value was found to be $\pm 1 \%$. Dielectric constant meter (DCL-01) (SES Instruments Pvt. Ltd., Roorkee, India) operating at $1 \mathrm{Mhz}$ frequency was used to measure dielectric constant $(\varepsilon)$ with an accuracy of $\pm 1 \%{ }^{18}$.

\section{Solvent Purification}

Dimethylsulfoxide (DMSO), (Merck) ${ }^{19}$ with boiling point $181-189^{\circ} \mathrm{C}$ was purified by repeated crystallisations. The crystallised solvent was further kept over $4 \mathrm{~A}^{\circ}$ molecular sieves for 2-3 days with occasional shaking and fractionated through a long vertical column under reduced pressure and middle fraction with density $1.0960 \mathrm{~g} \mathrm{~cm}^{-3}$ and viscosity $1.990 \mathrm{mPa}$.s was collected and stored. Pyridine (Py), (Merck) ${ }^{19}$ with boiling point of $114-115^{\circ} \mathrm{C}$ was refluxed over $\mathrm{KOH}$ for $3 \mathrm{~h}$ and was distilled at atmospheric pressure and middle fraction with density $0.9786 \mathrm{~g} \mathrm{~cm}^{-3}$ and viscosity $0.881 \mathrm{mPa}$.s was collected and stored.

Table 1: Source and Purity of Solvents

\begin{tabular}{|c|c|c|c|c|c|c|c|c|}
\hline Solvent & Provenance & CAS-No & $\begin{array}{l}\text { Molecular } \\
\text { Formula }\end{array}$ & $\begin{array}{l}\text { Molar Mass } \\
\qquad\left(\mathrm{g} \mathrm{mol}^{-1}\right)\end{array}$ & Grade & $\begin{array}{l}\text { Densitya } \\
\left(\mathrm{g} \mathrm{cm}^{-3}\right)\end{array}$ & $\begin{array}{c}\text { Mass Fraction } \\
\text { Purity }\end{array}$ & $\begin{array}{l}\text { Water } \\
\text { Content }\end{array}$ \\
\hline Dimethylsulfoxide (DMSO) & E.Merck & $67-68-5$ & $\mathrm{C}_{2} \mathrm{H}_{6} \mathrm{OS}$ & 78.13 & ACS & 1.0953 & $0.998^{\mathrm{b}}$ & $0.0002^{c}$ \\
\hline Pyridine (Py) & E.Merck & $110-86-1$ & $\mathrm{C}_{5} \mathrm{H}_{5} \mathrm{~N}$ & 79.1 & ACS, Reag. & 0.9786 & $0.995^{b}$ & $0.0001^{\mathrm{c}}$ \\
\hline
\end{tabular}

${ }^{a}$ density at $298 \mathrm{~K}$ by Anton Parr density meter (DSA $5000 \mathrm{M}$ ) with a precision of $0.0004 \mathrm{~g} \mathrm{~cm}^{-3}$

'From Gas Chromatography Analysis

'Karl-Fischer Titration Method 
Preparation of Tetrabutylammonium perchlorates and Tetrabutylammonium tetraphenyl borate

Tetrabutylammonium tetraphenylborate $\left(\mathrm{Bu}_{4} \mathrm{NBPh}_{4}\right)$ was prepared by mixing the aqueous solutions of tetrabutylammonium bromide $\left(\mathrm{Bu}_{4} \mathrm{NBr}\right)$ and sodium tetraphenylborate $\left(\mathrm{NaBPh}_{4}\right)$ in $1: 1$ molar ratio. The white powder of $\mathrm{Bu}_{4} \mathrm{NBPh}_{4}$ precipitated out which was filtered and dissolved in acetone and again was precipitated on adding distilled water in excess. Process of precipitation was repeated twice. The salt was dried under vacuum at $60^{\circ} \mathrm{C}$ over $\mathrm{P}_{2} \mathrm{O}_{5}$ for 2 days. Purity was checked by measuring its melting point. The measured melting point was $222-224^{\circ} \mathrm{C}$ which agreed well with the reported value of $223-225^{\circ} \mathrm{C}^{19}$. Tetrabutylammonium perchlorate $\left(\mathrm{Bu}_{4} \mathrm{NClO}_{4}\right)$ was prepared by prepared by dissolving silver perchlorate monohydrate $\left(\mathrm{AgClO}_{4} \cdot \mathrm{H}_{2} \mathrm{O}\right)$ (sisco research laboratories) and tetrabutyl ammonium bromide $\left(\mathrm{Bu}_{4} \mathrm{NBr}\right)$ in aqueous acetone mixtures in 1:1 molar ratio. After mixing, the solution was filtered, concentrated by evaporation and again filtered and heated till salt separated out. The process of dissolving in acetone and recrystallizing was repeated twice and salt was dried under $\mathrm{P}_{2} \mathrm{O}_{5}$ for 2 days at $60^{\circ} \mathrm{C}$.

\section{Theory}

The Isentropic compressibility $\left(\mathrm{K}_{\mathrm{s}}\right)$ and Acoustic parameters like Acoustic impedance $(\mathrm{Z})$, Free volume $\left(\mathrm{V}_{\mathrm{f}}\right)$, Absorption coefficient $\left(A b_{\text {scoeff }}\right)$, Internal pressure $(\pi \mathrm{i})$, Gibb's free energy $(\Delta G)$, Relaxation time $(\tau)$, Rao's constant $\left(R_{m}\right)$ Intermolecular free length $\left(\mathrm{L}_{\mathrm{f}}\right)$, Wada's constant $(\mathrm{w})$, and Entropy $(\mathrm{H})$ was calculated using experimental velocities, viscosities and densities at three different temperatures $(298 \mathrm{~K}, 308 \mathrm{~K}$ and $318 \mathrm{~K}$ ) and 1 atmospheric pressure. measured by ${ }^{20}$

Isentropic compressibility $\left(\mathrm{k}_{\mathrm{s}}\right)$ was

$$
\kappa_{s}=\frac{1}{\rho \times u^{2}}
$$

where $\rho$ denotes density and $u$, ultrasonic velocity.

The acoustic impedance $(Z)$ of a medium was calculated by a relation. ${ }^{21}$
$Z=\rho \times u$

Intermolecular free length $\left(\mathrm{L}_{f}\right)^{21}$ between the molecules in the liquid state was determined by the relation.

$\mathrm{L}_{\mathrm{f}}=\mathrm{K} \sqrt{ } \mathrm{K}_{\mathrm{s}}$

Where $\mathrm{K}$ is constant whose value is taken as $1.995 \times 10^{-6}$ at $298 \mathrm{~K}, 2.095 \times 10^{-6}$ at $308 \mathrm{~K}$ and $2.115 \times 10^{-6}$ at $318 \mathrm{~K}$.

Relaxation time $(\tau)$ was using the following relationship. ${ }^{22}$

$\tau=\frac{4 \eta}{3 \kappa_{s}}$

where $\eta$ is the viscosity coefficient.

Free Volume $\left(\mathrm{V}_{\mathrm{f}}\right)$ has been calculated from the relation. ${ }^{21}$

$\mathrm{V}_{\mathrm{f}}=\left(\frac{\mathrm{M}_{\text {eff. }} \mathrm{u}}{\mathrm{K} \eta}\right)^{3 / 2}$

Where, $M_{\text {eff }}$ is the effective molecular mass, $\mathrm{K}$ is temperature dependent constant.

Absorption coefficient $\left(A b_{\text {scoeff }}\right)$ of the medium was found by a relation. ${ }^{21}$

$\mathrm{Abs}_{\mathrm{coeff}}=\frac{8 \pi^{2} \eta}{3 \rho \times \mathrm{u}^{2}}$

The internal pressure $(\pi \mathrm{i})$ was calculated by the relation. ${ }^{21}$

$\pi_{\mathrm{i}}=\mathrm{bRT}\left(\frac{\mathrm{K} \cdot \eta}{\mathrm{u}}\right)^{1 / 2}\left(\frac{\rho^{2 / 3}}{M_{\mathrm{eff}}}\right)$

Where $b$ refers to cubic packing of solvent taken to be equal to $2, \mathrm{~K}$ is dimensionless constant having a value $4.281 \times 10^{9}, \mathrm{~T}$ is absolute temperature, $\eta$ is viscosity in $\mathrm{Nm}^{-2} \mathrm{~s}, \mathrm{R}$ is gas constant, $u$ is ultrasonic velocity in $\mathrm{m} \mathrm{s}^{-1}, \mathrm{M}_{\text {eff }}$ is effective molecular weight and $\rho$ is density in $\mathrm{kg} \mathrm{m}^{-3}$.

Gibbs free energy $(\Delta \mathrm{G})$ was determined from acoustic relaxation time $(\tau){ }^{20}$

$\Delta \mathrm{G}=\mathrm{RT} \ln \left(\frac{\mathrm{K} \cdot \mathrm{T} \cdot \tau}{\hbar}\right)$ 
Where $\mathrm{K}$ is Boltzmann constant, $\hbar$ is planck constant and $\tau$ is relaxation time.

Molar sound velocity or Rao's constant $\left(\mathrm{R}_{\mathrm{m}}\right)$ has been evaluated from the relation. ${ }^{20}$

$$
\mathrm{R}_{\mathrm{m}}=\frac{\mathrm{M}_{\text {eff. }}}{\rho} \cdot \mathrm{u}^{1 / 3}
$$

Wada's constant (w) has been calculated using isentropic compressibility values..$^{20}$

$\mathrm{W}=\left(\kappa_{\mathrm{s}}\right)^{-1 / 7} \cdot \frac{\mathrm{M}_{\mathrm{eff}}}{\rho}$ relation.

Enthalpy $(\mathrm{H})$ has been calculated by the

$$
\begin{aligned}
& \mathrm{H}=\pi_{\mathrm{i}} \times \mathrm{V}_{\mathrm{m}} \\
& \text { where } \mathrm{V}_{\mathrm{m}}=\frac{\mathrm{M}_{\mathrm{eff}}}{\rho}
\end{aligned}
$$

\section{RESULTS AND DISCUSSIONS}

In the present studies the Isentropic compressibility $\left(\mathrm{K}_{\mathrm{s}}\right)$ and Acoustic parameters like Acoustic impedance ( $Z$ ), Free volume $\left(\mathrm{V}_{\mathrm{f}}\right)$, Absorption coefficient $\left(\mathrm{Ab}_{\text {scoeff }}\right)$, Internal pressure $\left(\pi_{i}\right)$, Gibb's free energy $(\Delta G)$, Relaxation time $(\tau)$, Rao's constant $\left(R_{m}\right)$, Intermolecular free length $\left(L_{f}\right)$, Wada's constant $(w)$, and Entropy $(H)$ was calculated using experimental velocities, viscosities and densities at three different temperatures (298K, $308 \mathrm{~K}$ and $318 \mathrm{~K}$ ) and 1 atmospheric pressure for $\mathrm{Bu}_{4} \mathrm{NClO}_{4}$ and $\mathrm{Bu}_{4} \mathrm{NBPh}_{4}$ in a binary mixtures of DMSO+Py at 0, 20, 40,60, 80 and $100 \mathrm{~mol} \%$ composition of $\mathrm{Py}$.

Table 2: Density ( $\rho)$, viscosity $(\eta)$, and ultrasonic velocity (u) for DMSO and Py at 298K

\begin{tabular}{lcccccc}
\hline Solvents & \multicolumn{2}{c}{$\rho /\left(\mathrm{g} \mathrm{cm}^{-3}\right)$} & \multicolumn{2}{c}{$\eta /(\mathrm{mPa} \mathrm{s})$} & \multicolumn{2}{c}{$\mathrm{u} /\left(\mathrm{m} \mathrm{s}^{-1}\right)$} \\
& Expt. & Lit. & Expt. & Lit. & Expt. & Lit. \\
\hline DMSO & \multirow{2}{*}{1.0953} & $1.09530^{18} ;$ & 1.99 & $2.012^{24} ;$ & \multirow{2}{*}{1486.49} & $1486.74^{19}$ \\
& & $1.09533^{19} ;$ & & $1.99^{18} ;$ & & \\
Py & & $1.09537^{23}$ & & & & \\
& \multirow{2}{*}{0.9786} & $0.9782^{19,25}$ & 0.88 & $0.88^{19} ;$ & 1418.14 & $1419.2^{19}$ \\
\hline
\end{tabular}

Table 3: Density $(\rho)$, Viscosity $(\eta)$ and ultrasonic velocity (u) for DMSO+Py binary mixture at various temperaturesa

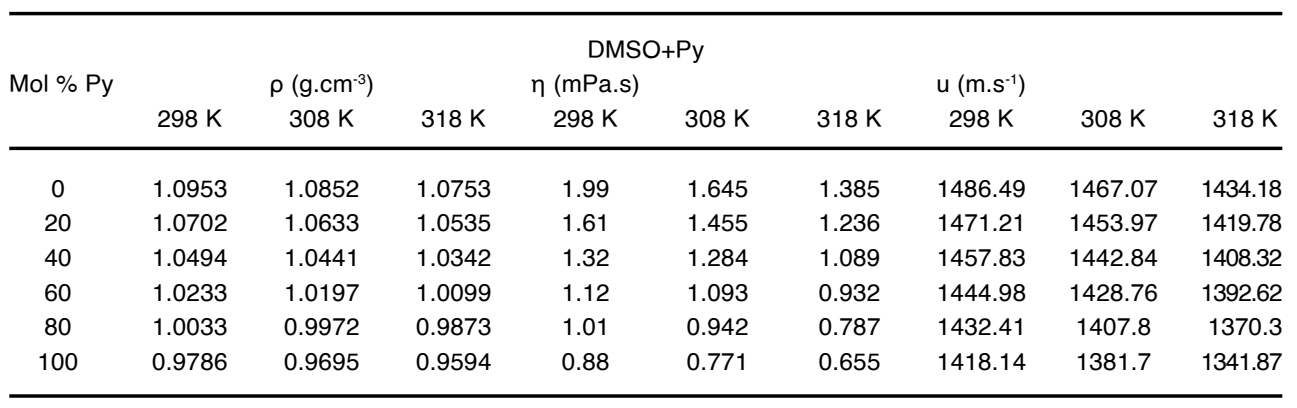

aRef: ${ }^{19}$

The previous investigations of the solvation of $\mathrm{Me}_{4} \mathrm{~N}^{+}, \mathrm{Et}_{4} \mathrm{~N}^{+}, \mathrm{Pr}_{4} \mathrm{~N}^{+}$and $\mathrm{Bu}_{4} \mathrm{~N}^{+}$ ions are limited to 2-methoxyethanol, water ${ }^{27}$, $\mathrm{N}, \mathrm{N}$-dimethylformamide, acetonitrile, DMSO and $\mathrm{n}$-butyronirile and their binary mixtures ${ }^{28,29}$. Since there is a limited data available for DMSO and Py, it is expected to be good solvents for a comparative study of the solvation behaviour of tetraalkylammonium ions. As evident from Tables $4-9$, with the increase in the concentration of electrolytes $\left(\mathrm{Bu}_{4} \mathrm{NBPh}_{4}, \mathrm{Bu}_{4} \mathrm{NClO}_{4}\right)$, the density shows an increase in trend and moreover ultrasonic velocity (u) increases. Increment in the ultrasonic velocity is related to compactness of medium as the number of molecules per unit volume increases, thus the medium becomes denser. It has been further supported by the fact that the electrolytes show two types of behaviour either structure maker or structure breaker. Increase in ultrasonic velocity corresponds to structure making tendency of electrolytes and decrease in velocity refers to structure breaking tendency. 
KAUR., Orient. J. Chem., Vol. 37(3), 722-734 (2021)

\begin{tabular}{|c|c|c|c|c|c|c|}
\hline 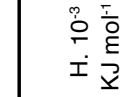 & 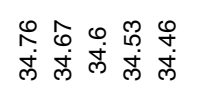 & 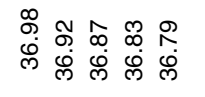 & 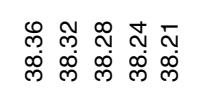 & 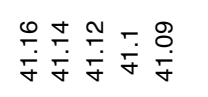 & 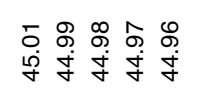 & 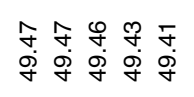 \\
\hline 3 & 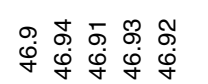 & 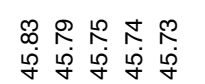 & 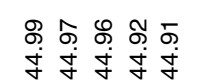 & 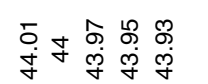 & 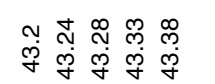 & 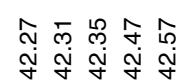 \\
\hline$\check{\Upsilon}^{\varepsilon}$ & 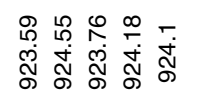 & 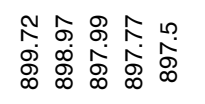 & 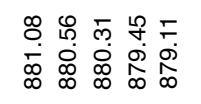 & 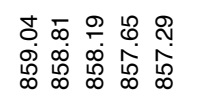 & 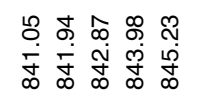 & 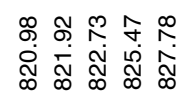 \\
\hline 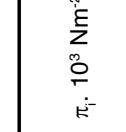 & 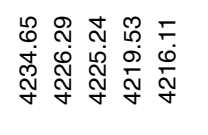 & 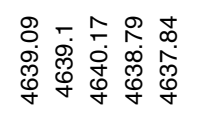 & 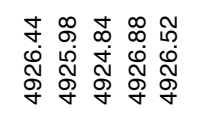 & 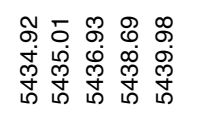 & 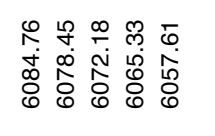 & 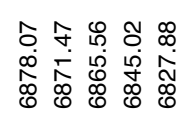 \\
\hline$\stackrel{\circ}{\circ} \stackrel{\bar{\partial}}{\underline{\varepsilon}}$ & 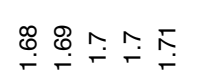 & 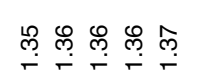 & 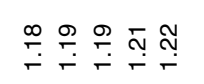 & ח & 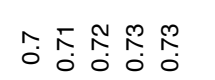 & 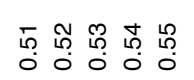 \\
\hline 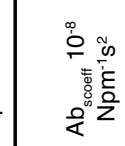 & 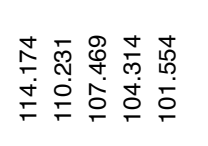 & 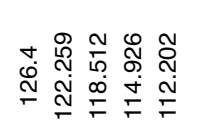 & 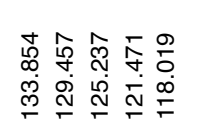 & 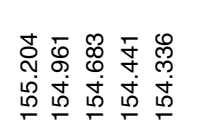 & 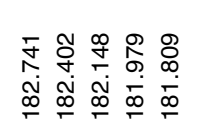 & 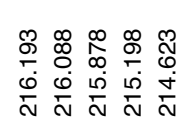 \\
\hline 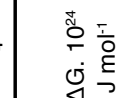 & 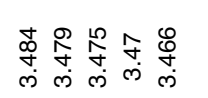 & 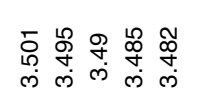 & 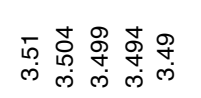 & 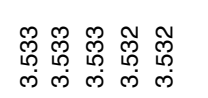 & 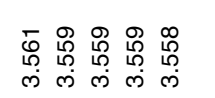 & 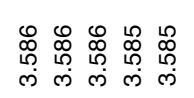 \\
\hline 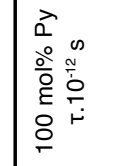 & 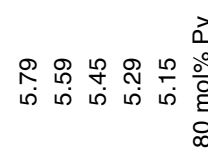 & (1) & 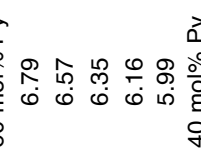 & 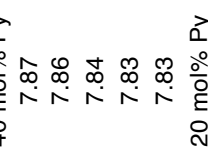 & 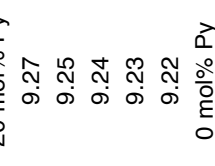 & $\begin{array}{l}\infty \\
\infty \\
\infty \\
\stackrel{\infty}{\infty}\end{array}$ \\
\hline$x^{4}$ & $\begin{array}{l}\infty \\
\infty \\
0\end{array}$ & 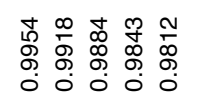 & 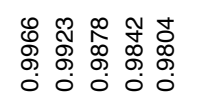 & 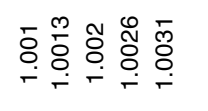 & 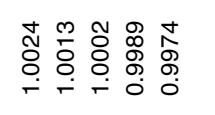 & 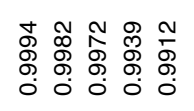 \\
\hline $\begin{array}{l}\varepsilon \\
\varepsilon \\
0 \\
\vdots \\
j \\
j\end{array}$ & 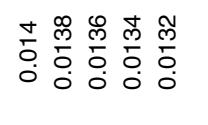 & 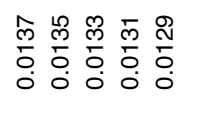 & 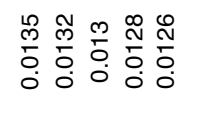 & 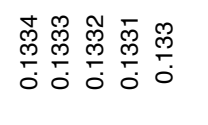 & 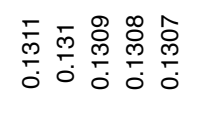 & 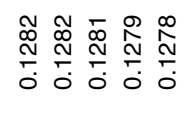 \\
\hline 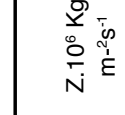 & 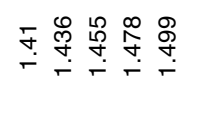 & 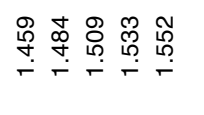 & 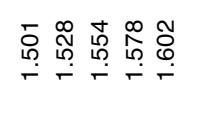 & 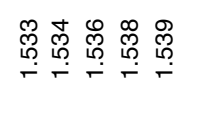 & 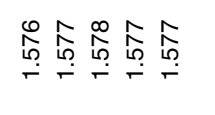 & 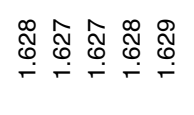 \\
\hline$x^{\circ}$ & 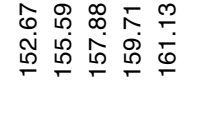 & 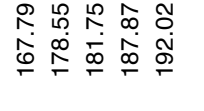 & 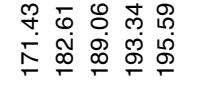 & 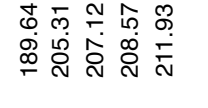 & 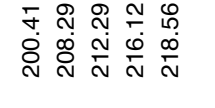 & 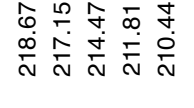 \\
\hline 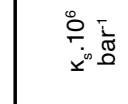 & 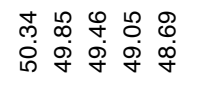 & 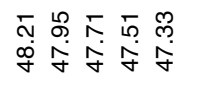 & 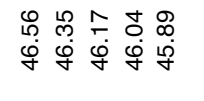 & 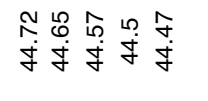 & 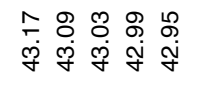 & 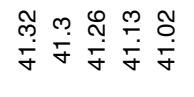 \\
\hline 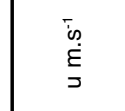 & 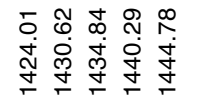 & 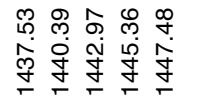 & 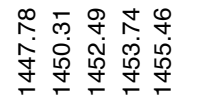 & 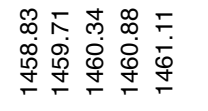 & 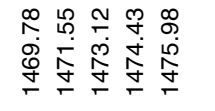 & 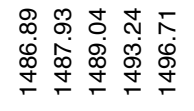 \\
\hline $\begin{array}{l}\stackrel{\infty}{0}_{0} \\
\dot{0} \\
0 \\
0\end{array}$ & 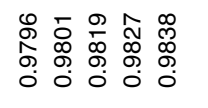 & 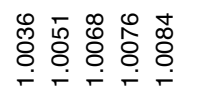 & 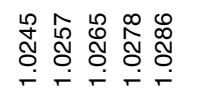 & 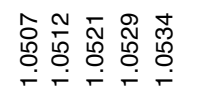 & 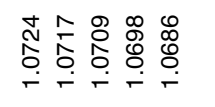 & 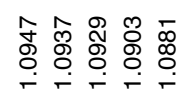 \\
\hline $\begin{array}{l}\text { ý } \\
\overline{0} \\
\underline{\varepsilon} \\
0 \\
0 \\
0\end{array}$ & 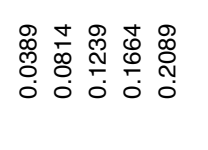 & 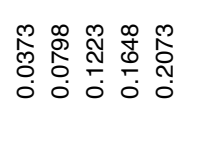 & 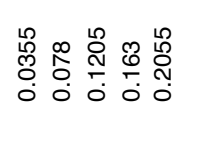 & $\begin{array}{l}\infty \\
\infty \\
0 \\
0\end{array}$ & 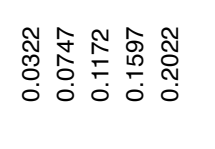 & 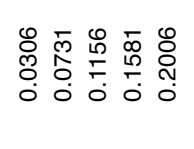 \\
\hline
\end{tabular}




\begin{tabular}{|c|c|c|c|c|c|c|}
\hline 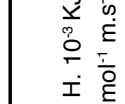 & 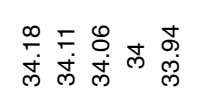 & 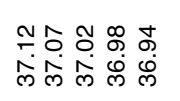 & 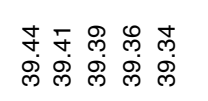 & 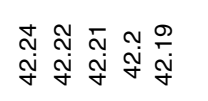 & 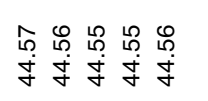 & 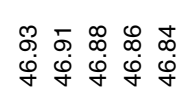 \\
\hline 3 & 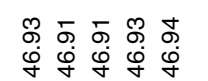 & 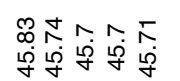 & 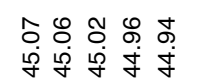 & 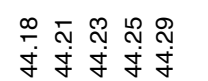 & 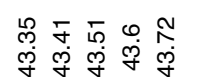 & 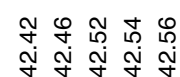 \\
\hline $\boldsymbol{\alpha}^{\mathrm{E}}$ & 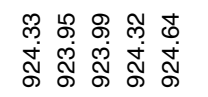 & 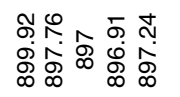 & 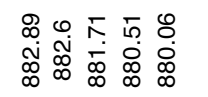 & 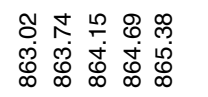 & 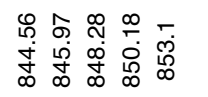 & 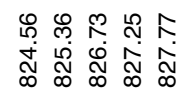 \\
\hline$K^{-}$ & 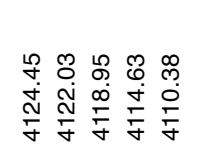 & 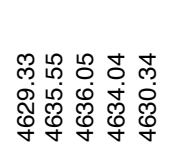 & 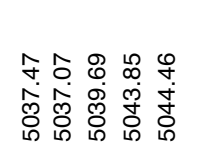 & 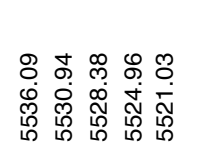 & 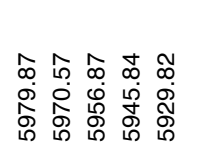 & 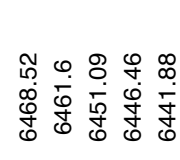 \\
\hline 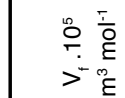 & 串 & 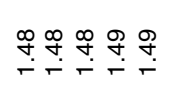 & 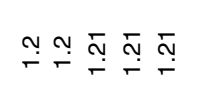 & 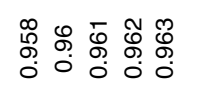 & 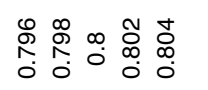 & 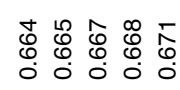 \\
\hline 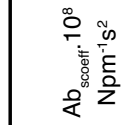 & 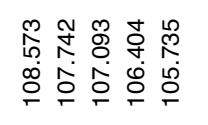 & 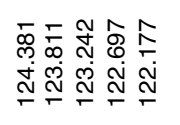 & 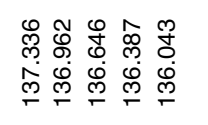 & 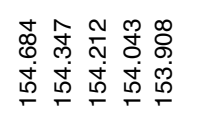 & 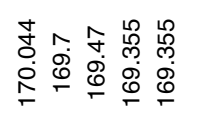 & 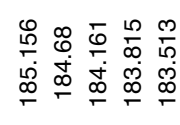 \\
\hline סे & 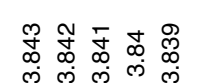 & 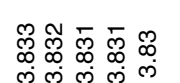 & 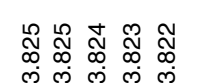 & 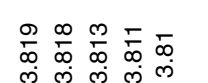 & 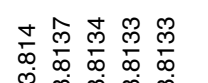 & 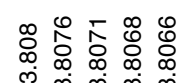 \\
\hline 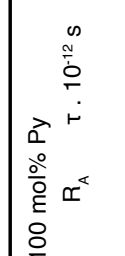 & 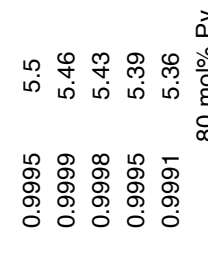 & 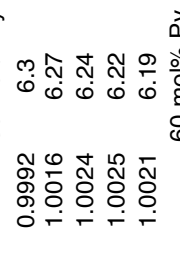 & 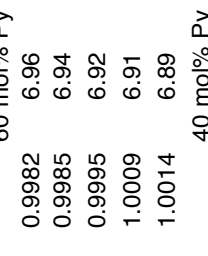 & 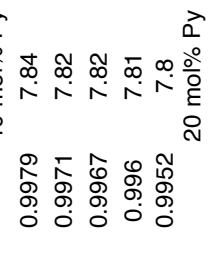 & 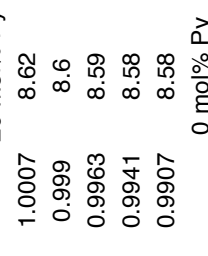 & 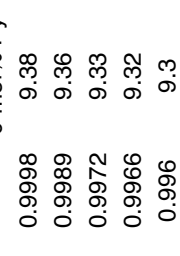 \\
\hline 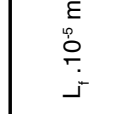 & 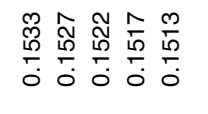 & 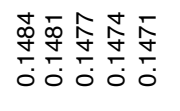 & 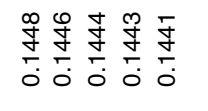 & 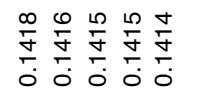 & 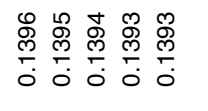 & 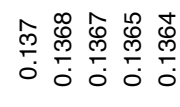 \\
\hline 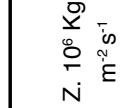 & 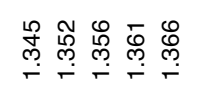 & 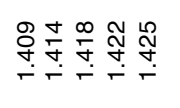 & 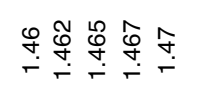 & 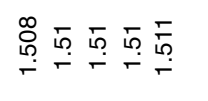 & ن & 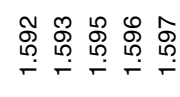 \\
\hline$x^{\hat{\phi}}$ & 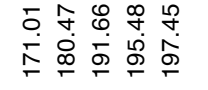 & 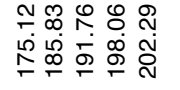 & 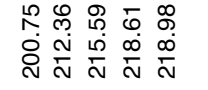 & 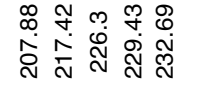 & 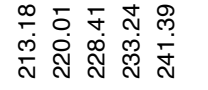 & 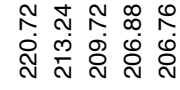 \\
\hline 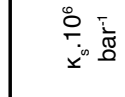 & 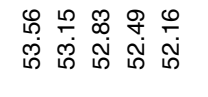 & 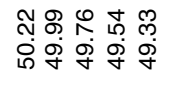 & 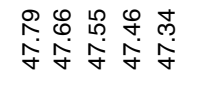 & 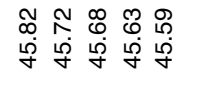 & 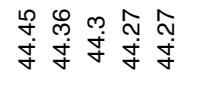 & 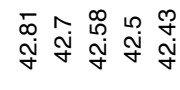 \\
\hline is & 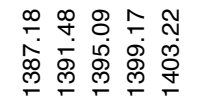 & 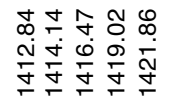 & 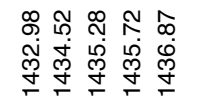 & 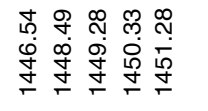 & 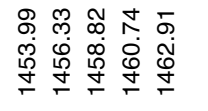 & 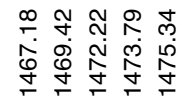 \\
\hline $\begin{array}{l}\stackrel{\infty}{\xi} \\
\dot{c} \\
\dot{0} \\
0\end{array}$ & 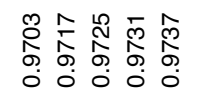 & 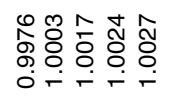 & 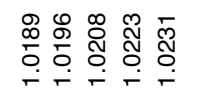 & 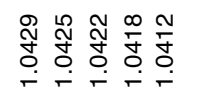 & 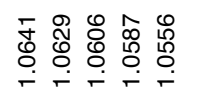 & 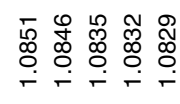 \\
\hline 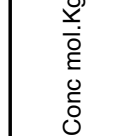 & 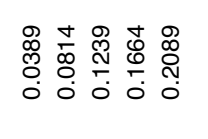 & 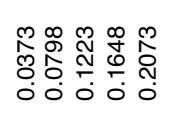 & 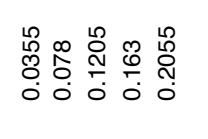 & $\begin{array}{l}\infty \\
\infty \\
0 \\
0\end{array}$ & 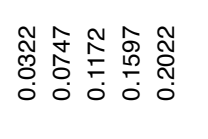 & 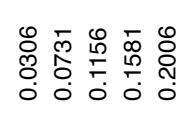 \\
\hline
\end{tabular}




\begin{tabular}{|c|c|c|c|c|c|c|}
\hline 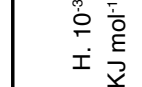 & 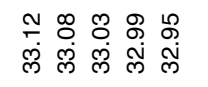 & 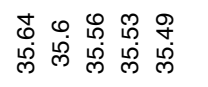 & 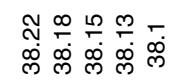 & 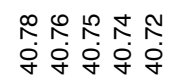 & 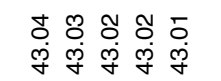 & 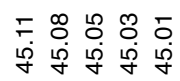 \\
\hline 3 & 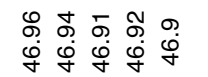 & 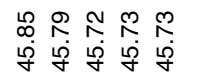 & 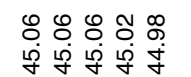 & 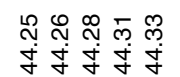 & 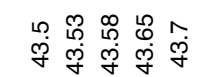 & 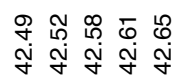 \\
\hline $\mathscr{\alpha}^{E}$ & 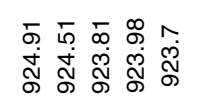 & 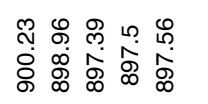 & 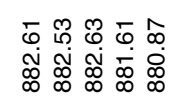 & 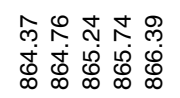 & 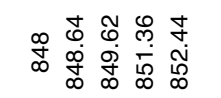 & 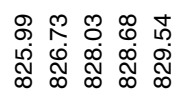 \\
\hline $\begin{array}{ll}-E \\
- \\
-2\end{array}$ & 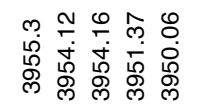 & 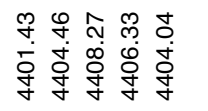 & 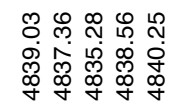 & 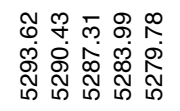 & 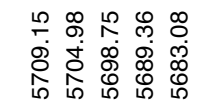 & 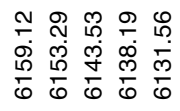 \\
\hline$\stackrel{\circ}{0}$ & 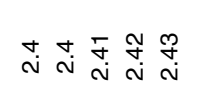 & 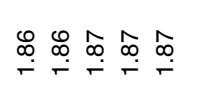 & $\stackrel{f}{\stackrel{f}{f}} \stackrel{f}{+} \stackrel{\infty}{=} \stackrel{\infty}{+} \stackrel{\infty}{=}$ & 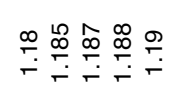 & 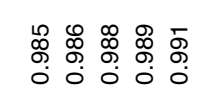 & 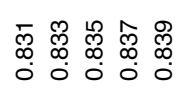 \\
\hline & 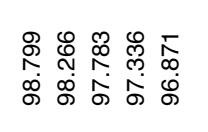 & 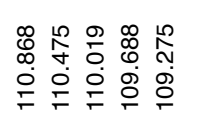 & 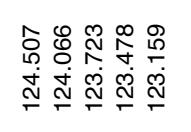 & 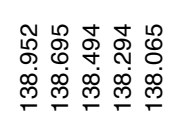 & 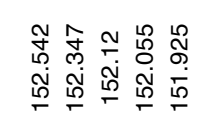 & 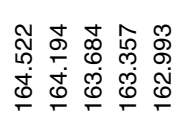 \\
\hline 六 & 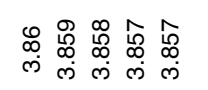 & 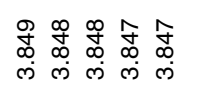 & 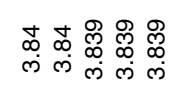 & 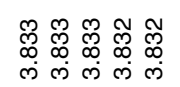 & 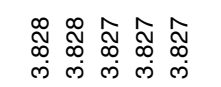 & 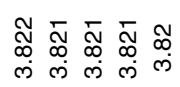 \\
\hline 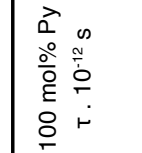 & б & 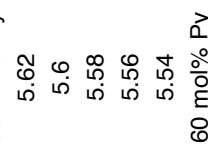 & 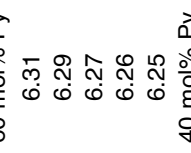 & סِمَ & 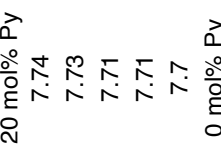 & 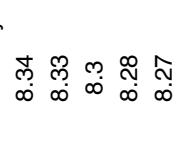 \\
\hline$\widetilde{\alpha}^{\alpha}$ & 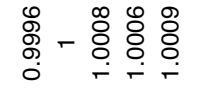 & 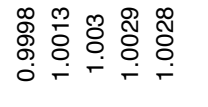 & 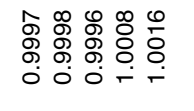 & 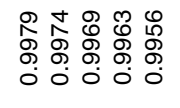 & 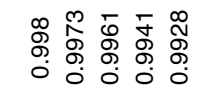 & 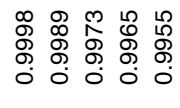 \\
\hline $\begin{array}{l}E \\
\qquad \\
0 \\
\vdots \\
j\end{array}$ & 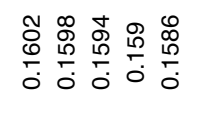 & 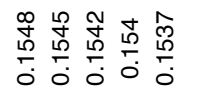 & 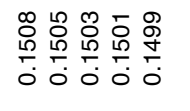 & 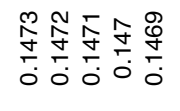 & 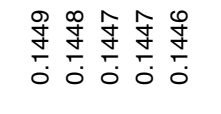 & 胥 \\
\hline 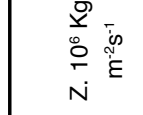 & 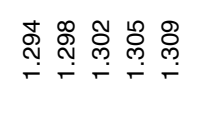 & 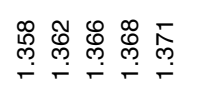 & 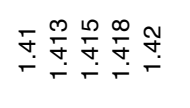 & 守 电 & 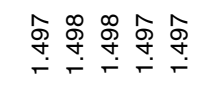 & 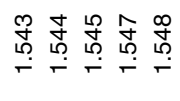 \\
\hline$x^{\bar{\phi}}$ & 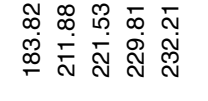 & 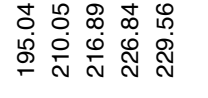 & 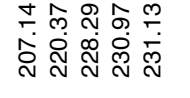 & 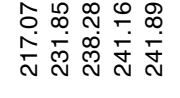 & 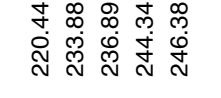 & 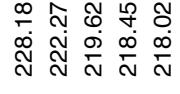 \\
\hline 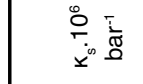 & 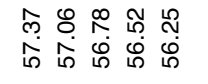 & 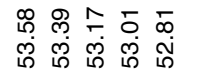 & 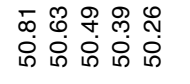 & 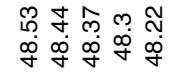 & 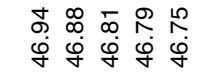 & 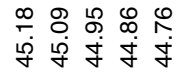 \\
\hline i & 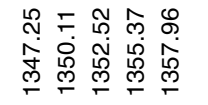 & 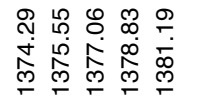 & 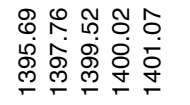 & 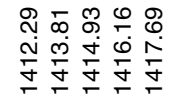 & 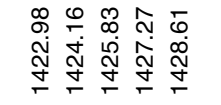 & 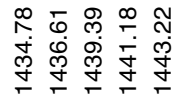 \\
\hline $\begin{array}{l}\ddot{E}_{0}^{0} \\
\dot{0} \\
0 \\
0\end{array}$ & 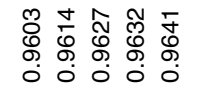 & 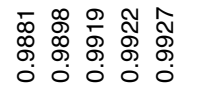 & mo & 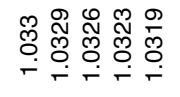 & 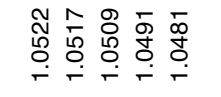 & 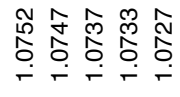 \\
\hline $\begin{array}{l}\vec{E} \\
0 \\
\tilde{O} \\
0\end{array}$ & 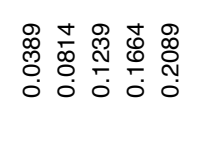 & 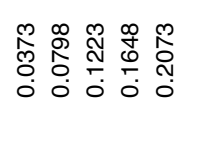 & 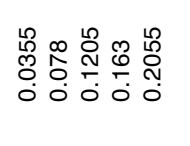 & 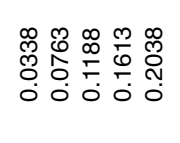 & 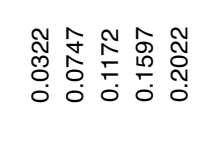 & 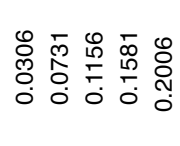 \\
\hline
\end{tabular}




\begin{tabular}{|c|c|c|c|c|c|c|}
\hline 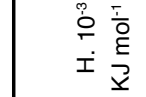 & 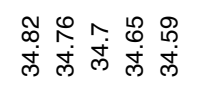 & 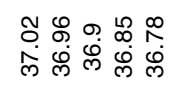 & 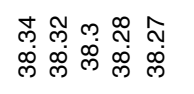 & 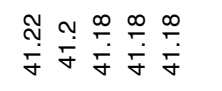 & 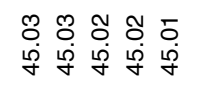 & 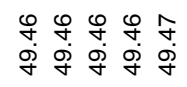 \\
\hline 3 & 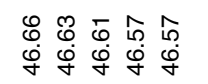 & 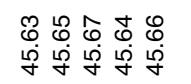 & 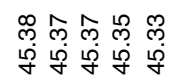 & 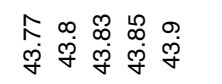 & 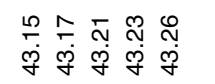 & 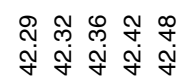 \\
\hline$\propto^{E}$ & 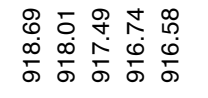 & 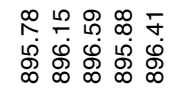 & 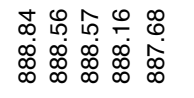 & 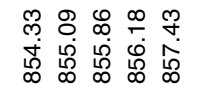 & 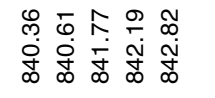 & 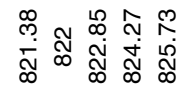 \\
\hline $\begin{array}{l}z \\
z \\
0 \\
0 \\
\dot{k} \\
k-1\end{array}$ & 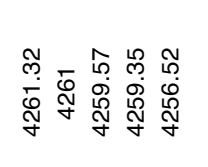 & 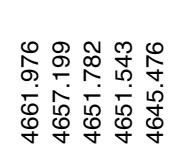 & 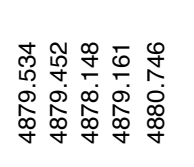 & 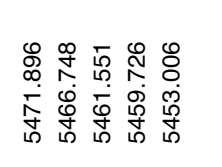 & 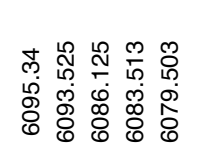 & 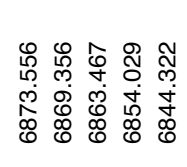 \\
\hline 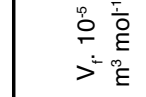 & 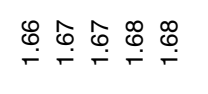 & 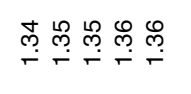 & 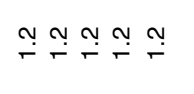 & 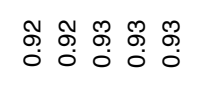 & 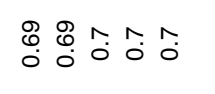 & 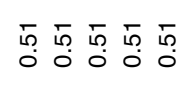 \\
\hline 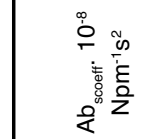 & 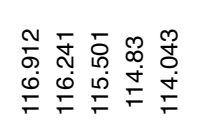 & 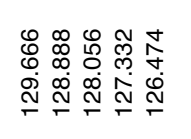 & 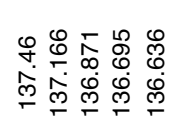 & 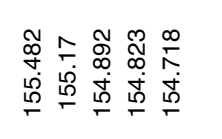 & 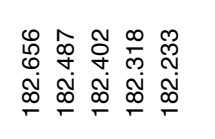 & 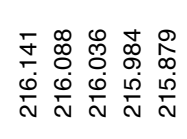 \\
\hline 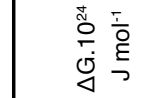 & 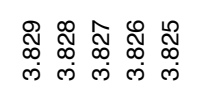 & 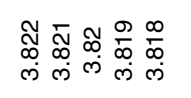 & 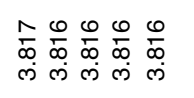 & 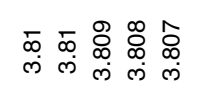 & 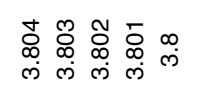 & 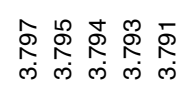 \\
\hline 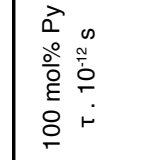 & 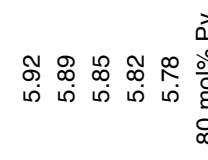 & 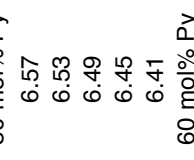 & 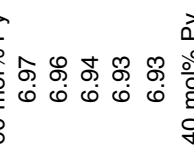 & 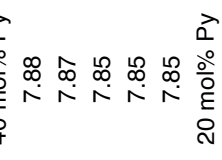 & 范 & 8 \\
\hline$x^{\alpha}$ & 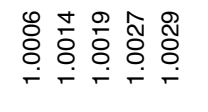 & 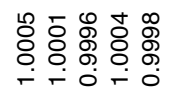 & 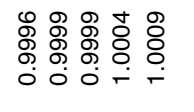 & 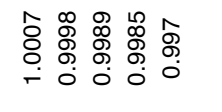 & 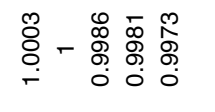 & 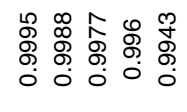 \\
\hline $\begin{array}{l}\varepsilon \\
\varepsilon \\
0 \\
0 \\
j=\end{array}$ & 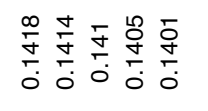 & 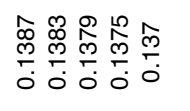 & 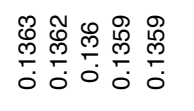 & 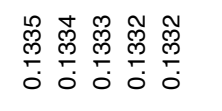 & 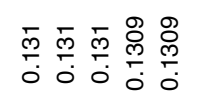 & 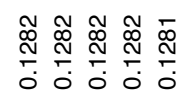 \\
\hline 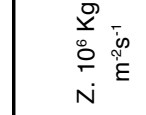 & 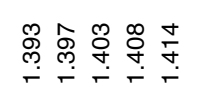 & 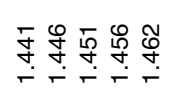 & 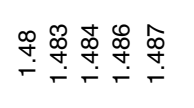 & 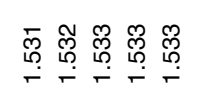 & 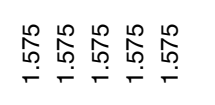 & 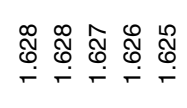 \\
\hline$x^{\hat{\theta}}$ & 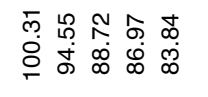 & 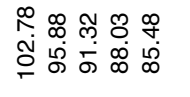 & 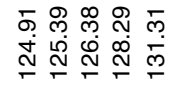 & 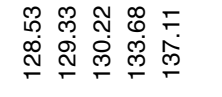 & 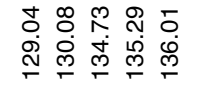 & 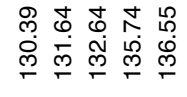 \\
\hline 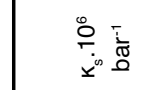 & 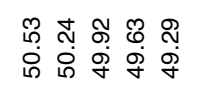 & 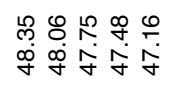 & 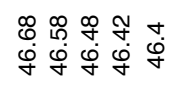 & 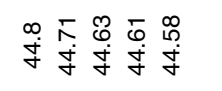 & 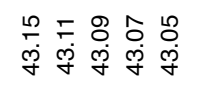 & 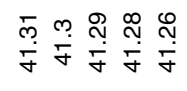 \\
\hline i & 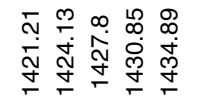 & 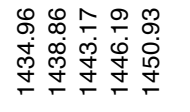 & 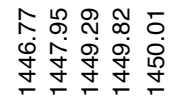 & 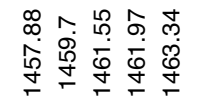 & 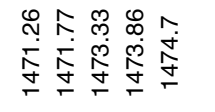 & 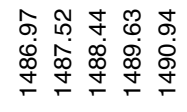 \\
\hline $\begin{array}{l}\sum_{0}^{0} \\
\dot{0} \\
0 \\
0 \\
0\end{array}$ & 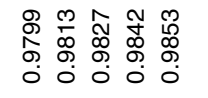 & 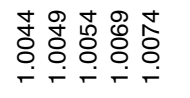 & 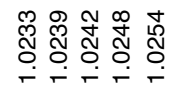 & 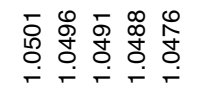 & 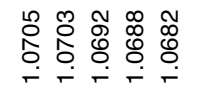 & 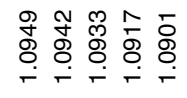 \\
\hline $\begin{array}{l}\overline{\bar{o}} \\
\dot{\bar{\varepsilon}} \\
\dot{0} \\
\dot{\bar{\sigma}}\end{array}$ & 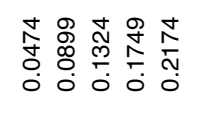 & 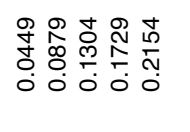 & 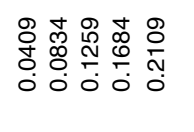 & 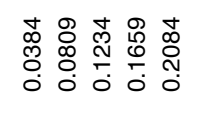 & 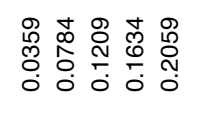 & 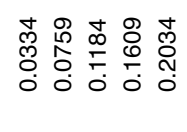 \\
\hline
\end{tabular}




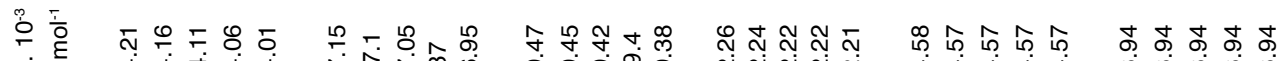

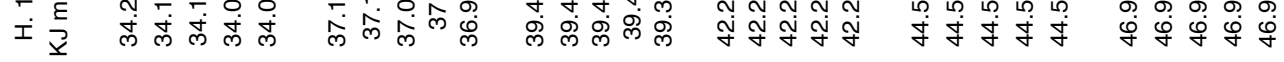

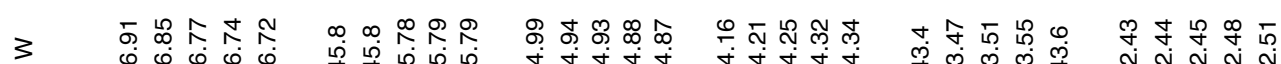

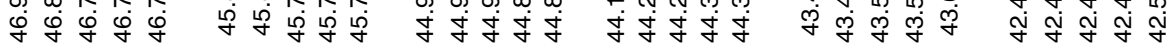

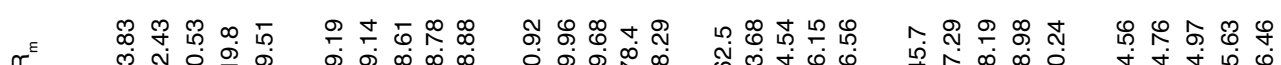

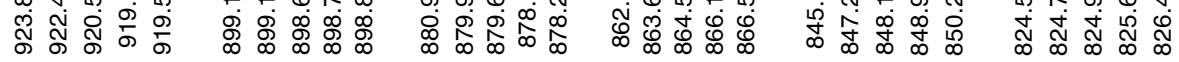

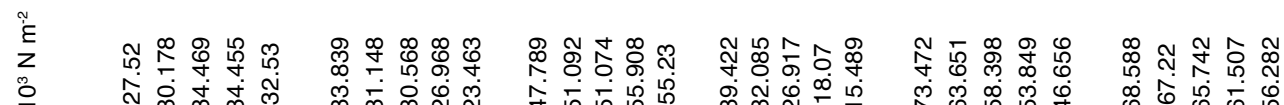

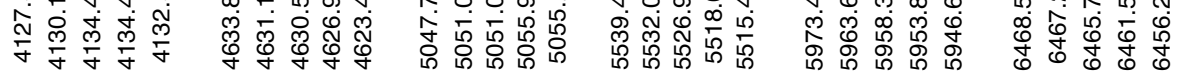

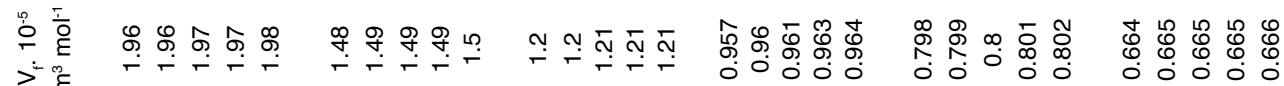

齐 喜产

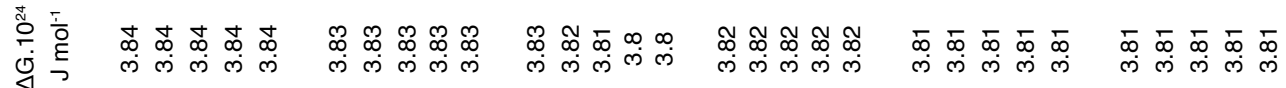

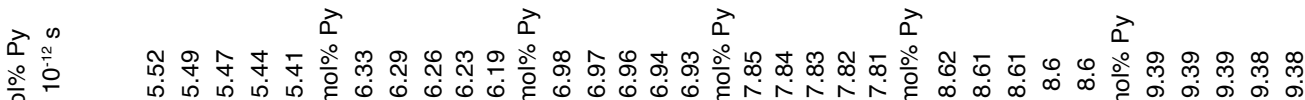
ํㅏㄹ

일

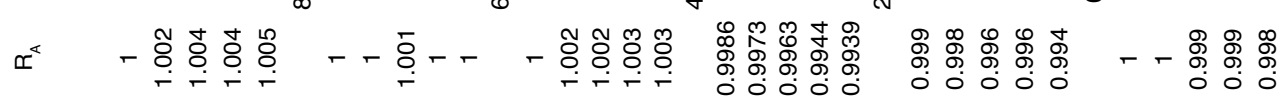

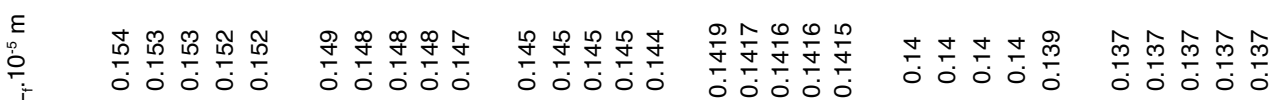

(1)

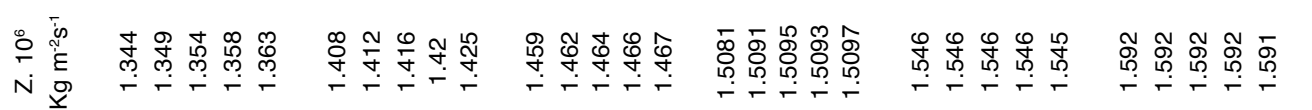

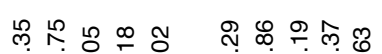

ㅁำ 중

(1)

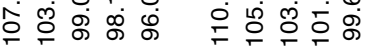

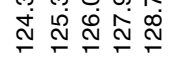

लिल

ชู่

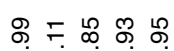

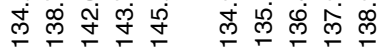

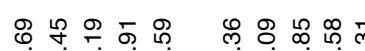

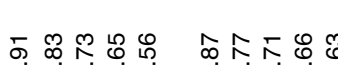

\&

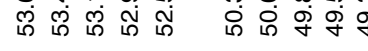

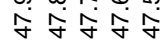

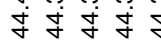

ปु่

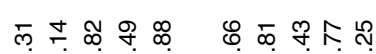

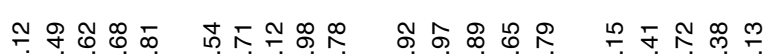

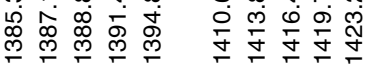

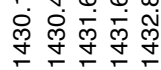

守守导守客

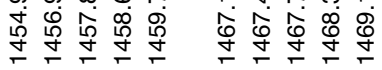

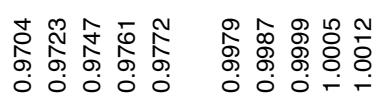

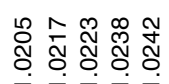

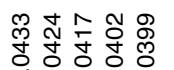

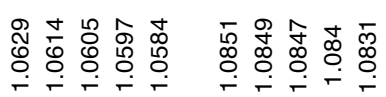

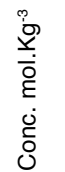

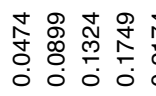

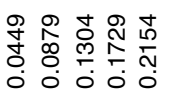

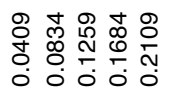

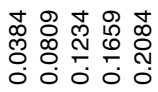

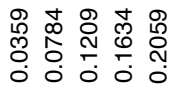

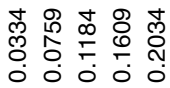




\begin{tabular}{|c|c|c|c|c|c|c|}
\hline 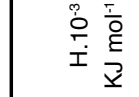 & 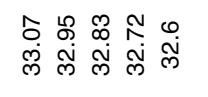 & 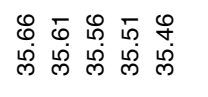 & 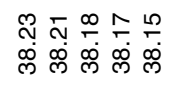 & 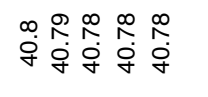 & 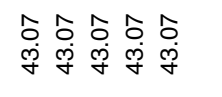 & 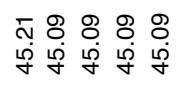 \\
\hline 3 & 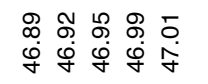 & 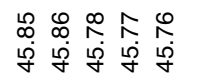 & 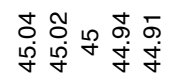 & 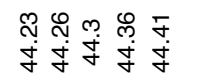 & 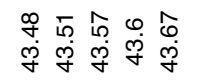 & 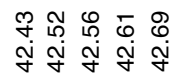 \\
\hline$\check{x}^{E}$ & 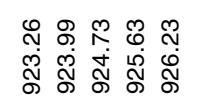 & 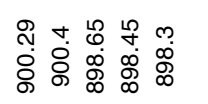 & 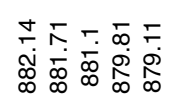 & 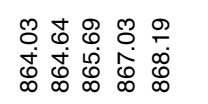 & 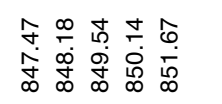 & 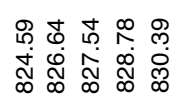 \\
\hline 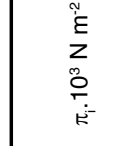 & 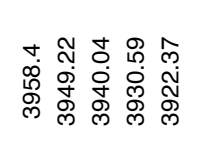 & 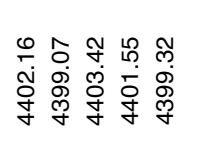 & 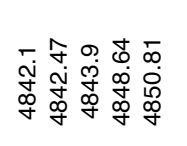 & 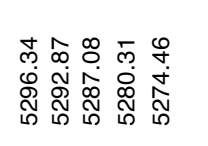 & 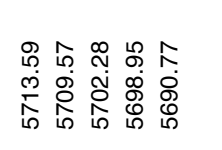 & 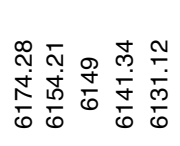 \\
\hline 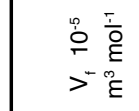 & 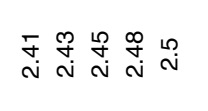 & 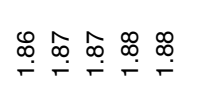 & 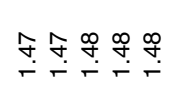 & 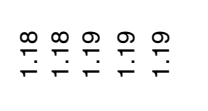 & 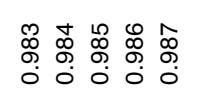 & 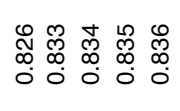 \\
\hline 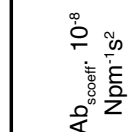 & 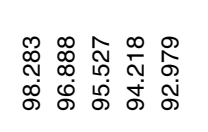 & 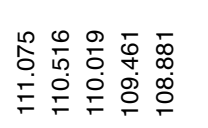 & 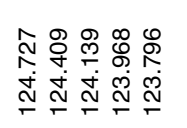 & 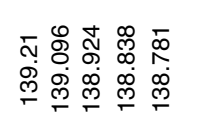 & 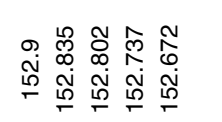 & 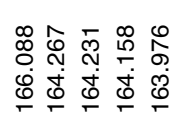 \\
\hline 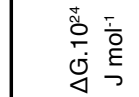 & 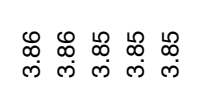 & 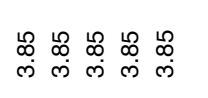 & 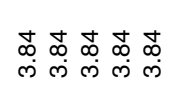 & 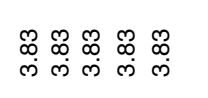 & 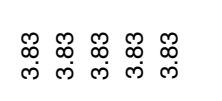 & 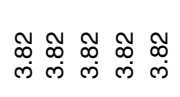 \\
\hline 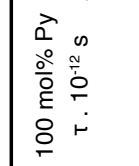 & 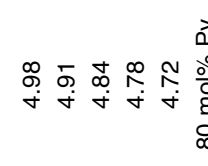 & ஜำ & 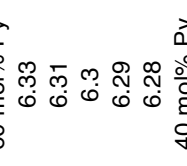 & 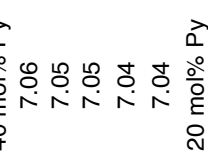 & 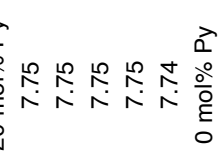 & ণ \\
\hline$\widetilde{\Upsilon}^{\varangle}$ & 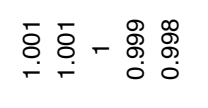 & 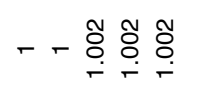 & - & 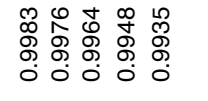 & 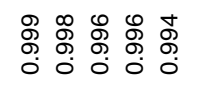 & 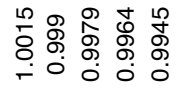 \\
\hline 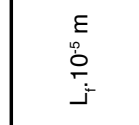 & 응 县 & 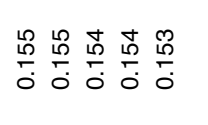 & 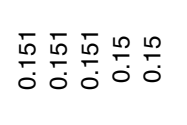 & 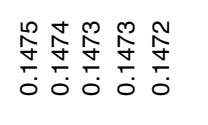 & 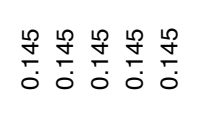 & 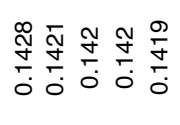 \\
\hline 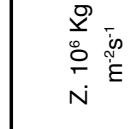 & 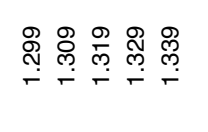 & 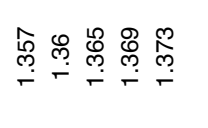 & 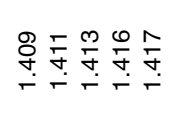 & 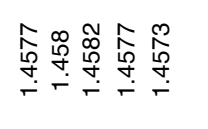 & 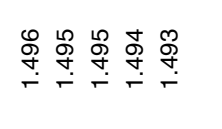 & 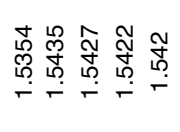 \\
\hline$x^{\bar{\phi}}$ & 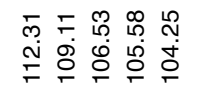 & 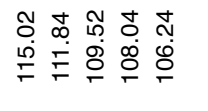 & 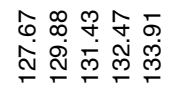 & 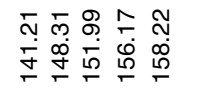 & 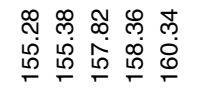 & 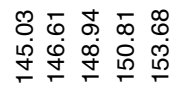 \\
\hline 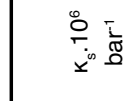 & 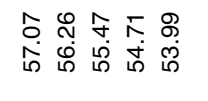 & 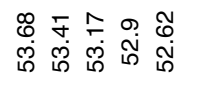 & 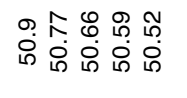 & 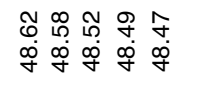 & 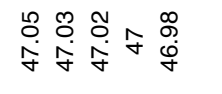 & 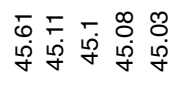 \\
\hline 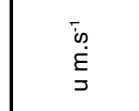 & 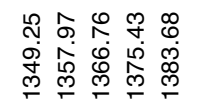 & 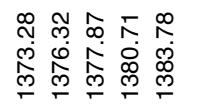 & 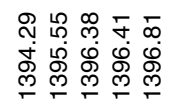 & 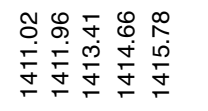 & 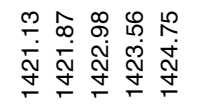 & 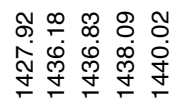 \\
\hline $\begin{array}{l}\stackrel{\infty}{E} \\
\dot{c} \\
\dot{0} \\
a\end{array}$ & 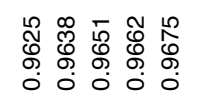 & 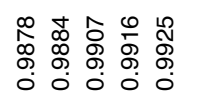 & 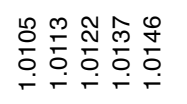 & 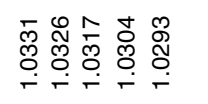 & 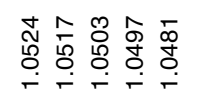 & 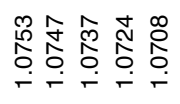 \\
\hline $\begin{array}{l}\frac{Y}{0} \\
\dot{0} \\
\dot{g} \\
\dot{0} \\
\dot{0} \\
\dot{0}\end{array}$ & 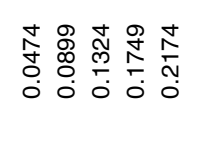 & 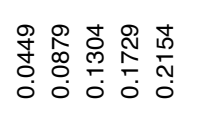 & 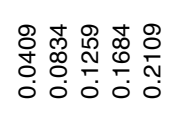 & 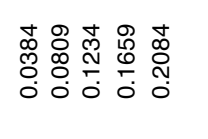 & 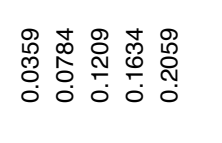 & 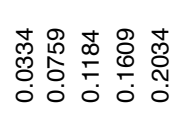 \\
\hline
\end{tabular}


Both $\mathrm{Bu}_{4} \mathrm{NBPh}_{4}, \mathrm{Bu}_{4} \mathrm{NClO}_{4}$, shows an increase in the ultrasonic velocity values at all the temperatures. This shows that molecular interactions are taking place in both the electrolytes. This increase in the molecular interactions with increase in the concentration of electrolytes in the solvent mixture may be due to solvent structural effects. Density measurements further supports these molecular interactions. With the increase in density, the interactions between solvent-solvent and solute-solvent is also increased. This could be attributed to the reduction in the free space per unit volume as more and more solute molecules occupied it. Isentropic compressibility $\left(\mathrm{K}_{\mathrm{s}}\right)$ shows the decreasing trend. When the solute molecule is added to the solvent, there are large numbers of solvent molecules available to interact and surround the incoming solute molecules. But on addition of more solute molecules in the solvent, the availability of solvent molecules in the bulk decreases. Because addition of an ion to the solvent attracts the solvent molecules towards itself, by pulling the molecules from surroundings. The electrostatic forces between incoming ions and the solvent molecule results in decrease in the availability of solvent molecules for next incoming ions. This process is defined as compressibility. Every solvent has a limit for compression called limiting compressibility. The solvent's compressibility is generally more than that of a solute and is inversely related to concentration. Ks decreases with increase in concentration because as the concentration of solutes increases, a larger portion of solvent molecules being electrostatic attract towards solute molecules and there is reduction in the amount of solvent molecules in the bulk which inversely decreases. $\mathrm{Bu}_{4} \mathrm{NBPh}_{4}$, $\mathrm{Bu}_{4} \mathrm{NClO}_{4}$, both shows the same decreasing trend at all compositions of $\mathrm{Py}$ solvents and trend is followed at high temperatures also.

The Acoustic Impedance ( $Z$ ), for the electrolyte is related to density and ultrasonic velocity of the medium. $Z$ values increases with the increase in the concentration of $\mathrm{Bu}_{4} \mathrm{NBPh}_{4}, \mathrm{Bu}_{4} \mathrm{NClO}_{4}$, at $298 \mathrm{~K}, 308 \mathrm{~K}$ and $318 \mathrm{~K}$. The value of $Z$ further increases when we move to DMSO rich regions than in Py rich regions. This behaviour suggests the possibility of molecular interactions between solutes and solvent through hydrogen bonding. Intermolecular free length $\left(L_{f}\right)$, decreases with increase in the concentration of electrolytes which indicates less number of solvent molecules available in the bulk but the value increases with the increase in temperature ${ }^{30}$. Thus intermolecular free length shows inverse relationships with the ultrasonic velocity. Lesser the intermolecular distance between the solvent and solute molecules, more will be the free length. Thus this leads to high ultrasonic velocity of the medium. Both $\mathrm{Bu}_{4} \mathrm{NBPh}_{4}, \mathrm{Bu}_{4} \mathrm{NClO}_{4}$, shows approximately same trend in the $\mathrm{L}_{\mathrm{f}}$ values.

Relative association $\left(R_{A}\right)$, is further influenced either by the breaking up of the solvent structures on addition of solutes or by the relative solvation of the solutes. The former trend refers to decrease in the $R_{A}$ values and latter corresponds to increase in the $R_{A}$ values. These values show no regular trend in both the electrolytes studied. These values were found to be maximum at 80 mol\% Py for $\mathrm{Bu}_{4} \mathrm{NBPh}_{4}$, which is greater than one. This indicates that $\mathrm{Bu}_{4} \mathrm{NBPh}_{4}$, is solvated to greater extent at $80 \mathrm{~mol} \% \mathrm{Py}$. But both in Pure Py or pure DMSO, the $R_{A}$ values are less than one which indicates the breaking up of the solvent structures with addition of solute molecules. This trend remains same at all the temperatures studied. But the $R_{A}$ values for $\mathrm{Bu}_{4} \mathrm{NClO}_{4}$ is positive and greater than one which indicates that for $\mathrm{Bu}_{4} \mathrm{NClO}_{4}$, shows more molecular interactions in DMSO as well as in Py at all compositions and at all three temperatures. Relaxation time $(\tau)$ decreases with increase in the concentration of $\mathrm{Bu}_{4} \mathrm{NBPh}_{4}, \mathrm{Bu}_{4} \mathrm{NClO}_{4}$, and shows a decreasing trend as we move to $P y$ rich regions. The relaxation time further shows a decreasing trend with increase in temperature. Relaxation time indicates the time taken by solute molecules to undergo any structural changes ${ }^{31,32}$. Gibbs free energy $(\Delta G)$ measures the close packing of molecules which might be due to some kind of bonding (hydrogen or van der wall) between electrolyte and the solvent molecules. The decrease in the trend of free energy values suggest that less time is required for the cooperation process or rearrangement of molecules in the solvent ${ }^{33}$. Values of absorption coefficient $\left(a / f^{2}\right)$ decreases with increase in the concentration of electrolytes and also decreases with the increase in the temperature. This indicates that the interaction in Py rich regions is less for both the electrolytes and same trend is followed at high temperature also ${ }^{34}$. Thus it shows greater molecular interaction in DMSO rich regions and Entropy $(\mathrm{H})$ follows the same pattern. 
Internal Pressure $\left(\pi_{i}\right)$ value decreases when the concentration of both $\mathrm{Bu}_{4} \mathrm{NBPh}_{4}, \mathrm{Bu}_{4} \mathrm{NClO}_{4}$, increases indicating that the molecular interactions is greater at lower concentration of solute and these interactions are more for DMSO than for $\mathrm{Py}^{35}$. It is also very interesting to see that free volume of the system increases as the internal pressure decreases. The higher value of free volume indicates weak solute-solute interactions and vice-versa. Decrease in the internal pressure might be attributed to the loosening of cohesive forces which leads to breaking up of solvent structure at Py rich regions for $\mathrm{Bu}_{4} \mathrm{NBPh}_{4}$ and $\mathrm{Bu}_{4} \mathrm{NClO}_{4}$. Rao constant $\left(\mathrm{R}_{\mathrm{m}}\right)$ and Wada constant $(\mathrm{W})$ did not show much change in the values with increase in the concentration of solute. But this increase in the Py rich region indicating the presence of higher number of molecules in same region which lead to compact packing of medium thus increasing the interaction.

\section{CONCLUSION}

Acoustic studies of tetrabutylammonium tetraphenylborate and tetrabutylammonium perchlorate in a mixture of dimethylsulfoxide and pyridine at $298 \mathrm{~K}, 308 \mathrm{~K}$ and $318 \mathrm{~K}$ were carried out for the present studies. Tetraalkylammonium salts exclusively find an application as electrolytes in developing super capacitors also known as electrical double layer capacitors (EDLCs). Results showed greater molecular interaction in DMSO rich regions for both $\mathrm{Bu}_{4} \mathrm{NBPh}_{4}$ and $\mathrm{Bu}_{4} \mathrm{NClO}_{4}$, at all temperatures. This increase in the molecular interactions with increase in the concentration of electrolytes in the solvent mixture may be attributed to solvent structural effects.

\section{ACKNOWLEDGMENT}

This research did not receive any specific grant from funding agencies in the public, commercial, or not-for-profit sectors.

\section{Conflict of interest}

The author declare that we have no conflict of interest.

\section{REFERENCES}

1. Gill, D.S.; Anand, H.; Pathania, V., Z. Phys. Chem., 2004, 218, 857-865.

2. Gill, D.S.; Rodehueser, L.; Rubini, P.; Delpuech, J. J., J. Chem. Soc. Faraday Trans., 1995, 91, 2307.

3. Gill, D.S.; Kemp, U.; Dölle, A.; Zeidler, M.D., Ind. J. Chem., 2001, 40A, 693-699.

4. Saravanakumar, K.; Baskaran, R.; Kubendran, T.R., Russ. J. Phys. Chem. A., 2012, 86(13), 1947-1952.

5. Bedare, G.R.; Bhandakkar, V.D.; Suryavanshi, B.M., Euro. J. Appl. Eng. Sci. Res., 2012, 1(1), 1-4.

6. Amrutia, R.R.; Mehta, N.M.; Karia, F.D.; Parsania. P.H., J. Sci. Ind. Res., 2006, 65, 905-911.

7. Praharaj, M.K.; Satapathy, A.; Mishra. P.; Mishra, S., J. Chem. Pharma. Res., 2013, 5(1), 49-56.

8. Bhandakkar, V.D.; Bhat, V.R.; Chimankarand, O.P.; Asole. A.W., Adv. Appl. Sci. Res., 2014, 5(2), 80-85.
9. Rawat, M.K.; Sangeeta., Ind. J. Pure and Appl. Phys., 2008, 46(3), 187-192.

10. Jasmine, E.; Rani, V.; Kannagi, K.; Padmavathy, R.; Radha, N., J. Bas. Appl. Phys., 2012, 1, 96-101.

11. Singh, P.K.; Bhatt, S. C., Appl. Phys. Res., 2010, 2(1), 35-41.

12. Tabhane, P.; Chimankar, O.P.; Dudhe, C.M.; Tabhane; V.A., Der. Chemica. Sinica., 2012, 3(4), 944-947.

13. Wadekar, M.P., J. Chem. Pharma. Res., 2013, 5(8), 37-41.

14. Kaur, B.; Juglan, K. C., J. Poly. Eng., 2013, 33, 851-856.

15. Rodrıguez,. H.; Brennecke, J. F., J. Chem. Eng. Data., 2006, 51, 2145-2155.

16. Del Grosso, V.A.; Mader, C.W., J. Acoust. Soc. Am., 1972, 52, 1442-46.

17. Gill, D.S.; Rohitash.; Anand, H.; Puri, J.K., J. Mol. Liq., 2002, 15, 98-99.

18. Pathania, V.; Kaur, M.; Vermani, B.K.; Veneeta,; Gill, D.S., J. Soln. Chem., 2021. https://doi.org/10.1007/s10953-021-01086-3 
19. Kaur, M., IOP Conf. Ser.: Mater. Sci. Eng., 2021, 1033, 012079.

20. Mehra, R.; Malav, B.B., Arab. J. Chem., 2013, DOI: 10.1016/j.arabjc.2013.07.018.

21. Godhani, D.R.; Dobariya, P.B.; Sanghani, A.M.; Mehta, J.P., Arab. J. Chem., 2012, 10, 422-430. http://dx.doi.org/10.1016/j. arabjc.2012.10.002.

22. Dash, U.N.; Roy, G.S.; Talukdar, M.; Moharatha, D., Ind. J. of Pure \& Appl. Phys., 2010, 48, 651-657.

23. Markarian, S.A.; Asatryan, A.M.; Zatikyan, A.L., J. Chem. Thermodyn., 2005, 37, 768-777.

24. Zhao, T.; Zhang, J.; Guo, B.; Zhang, F.; Sha, F.; Xie, X,; Wei, X., J. Mol. Liq., 2005, 207, 315-322.

25. Kinart, C.M.; Kinart, W.J.; Aewiklinska, A,; Kinart, Z., Phys. Chem. Chem. Phys., 2014, 52, 627-635.

26. Kijevcanin, M.L.; Zivkovic, E.M.; Djordjevic, B.D.; Radovic, R.; Jovanovic, J.; Serbanovic,
S., J. Chem. Thermodyn., 2013, 56, 49-56.

27. Gill, D.S.; Kumari, A.; Kumar, S.; Jauhar, S.P., Z Naturforsch., 2005, 60A, 70-74.

28. Kratochvil, B.; Yeager, H.L., Topic in Current Chemistry., 1972, 27, 1.

29. Anand, H.; Verma, R., Chem Sci Trans., 2018, 7(3), 488-498.

30. Shinde, B.R.; Jadhav, S.S.; Shinde, S.U.; Shengule, D.R.; Jadhav, K.M., J. Chem. Pharm. Res., 2011, 3(3), 432-438.

31. Sharma, P.; Chauhan, S.; Chauhan, M.S.; Syal, V.K., Ind J Pure and Appl Phys., 2008, 46, 839-843.

32. Ali, A.; Hyde, S.; Nain, A.K., Ind. J. Phys., 2000, 74(B), 63-67.

33. Naik, A.B.; Ind. J. Pure and Appl. Phys., 2015, 53, 27-34.

34. Kannappan, A.N.; Ali, S.J.A.; Mahaboob, A., Ind J Pure and Appl Phys., 2009, 47, 97.

35. Kolhe, R.K.; Bhosale, B.B., Int. J. Scient. And Resc. Pub., 2017, 7, 494-511. 